\title{
Naturalness and dark matter in a realistic intersecting D6-brane model
}

\author{
Waqas Ahmed, ${ }^{a}$ Lorenzo Calibbi, ${ }^{a}$ Tianjun Li, ${ }^{a, b, c}$ Shabbar Raza, ${ }^{d}$ Jia-Shu Niu ${ }^{a, b}$ \\ and Xiao-Chuan Wang ${ }^{e}$ \\ ${ }^{a}$ CAS Key Laboratory of Theoretical Physics, Institute of Theoretical Physics, \\ Chinese Academy of Sciences, Beijing 100190, P.R. China \\ ${ }^{b}$ School of Physical Sciences, University of Chinese Academy of Sciences, \\ Beijing 100049, P.R. China \\ ${ }^{c}$ School of Physical Electronics, University of Electronic Science and Technology of China, \\ Chengdu 610054, P.R. China \\ ${ }^{d}$ Department of Physics, Federal Urdu University of Arts, Science and Technology, \\ Karachi 75300, Pakistan \\ ${ }^{e}$ Department of Physics, Henan Normal University, \\ Xinxiang, Henan, 45300\%, P.R. China \\ E-mail: waqasmit@itp.ac.cn, calibbi@itp.ac.cn, tli@itp.ac.cn, \\ shabbar.raza@fuuast.edu.pk, jsniu@itp.ac.cn, xcwang@live.com
}

Abstract: We revisit a three-family Pati-Salam model with a realistic phenomenology from intersecting D6-branes in Type IIA string theory compactified on a $\mathbf{T}^{6} /\left(\mathbb{Z}_{2} \times \mathbb{Z}_{2}\right)$ orientifold, and study its naturalness in view of the current LHC and dark matter searches. We discuss spectrum and phenomenological features of this scenario demanding fine tuning better than $1 \%$. This requirement restricts the lightest neutralino to have mass less than about $600 \mathrm{GeV}$. We observe that the viable parameter space is tightly constrained by the requirements of naturalness and consistency with the observed dark matter relic density, so that it is fully testable at current and future dark matter searches, unless a non-thermal production mechanism of dark matter is at work. We find that $Z$-resonance, $h$-resonance, $A$-funnel and light stau/stop-neutralino coannihilation solutions are consistent with current LHC and dark matter constraints while the "well-tempered" neutralino scenario is ruled out in our model. Moreover, we observe that only Bino, Higgsinos, right-handed staus and stops can have mass below $1 \mathrm{TeV}$.

KEYwords: Strings and branes phenomenology, Supersymmetry Phenomenology

ARXIV EPRINT: 1711.10225 


\section{Contents}

1 Introduction 1

2 The realistic Pati-Salam model from intersecting D6-branes 3

3 Scanning procedure and constraints $\quad 4$

$\begin{array}{lll}4 & \text { Results and discussion } & 7\end{array}$

5 Summary and conclusions $\quad 11$

A Tuning of the high-energy parameters 12

\section{Introduction}

Despite the extensive searches performed at the Large Hadron Collider (LHC), no evidence for physics beyond the Standard Model (SM) has been found so far. Together with the observation of the Higgs boson, whose properties are within the uncertainties in good agreement with the SM predictions, this challenges the extensions of the SM that have been proposed to provide a natural explanation of the hierarchy between the electroweak symmetry breaking (EWSB) scale and the Planck scale. In particular, the limits set by the searches performed by the ATLAS and CMS collaborations on the mass of possible supersymmetric partners of the SM particles and the measured mass of the Higgs boson, $m_{H} \simeq 125 \mathrm{GeV}$, push low-energy supersymmetry (SUSY) — once the most popular attempt to solve the gauge hierarchy problem - into the range of fine tuning worse than the percent level, at least in the simplest SUSY-breaking scenarios. Therefore, we think that, before giving up naturalness as a motivation for new physics, it is worth to survey possible exceptions to the above conclusion in the attempt of finding non-minimal and comparatively natural solutions. Indeed, several examples of such kind have been discussed in recent literature [1-41]. In particular, an interesting scenario has been recently proposed, which was called 'Super-Natural' SUSY [42-44]. In this framework, no residual electroweak finetuning is left in the Minimal Supersymmetric Standard Model (MSSM) in presence of noscale supergravity boundary conditions [45-49] and Giudice-Masiero (GM) mechanism [50], despite a relatively heavy spectrum. ${ }^{1}$

Apart from the gauge hierarchy problem, the most compelling motivation for new physics at energies accessible at the LHC is probably given by the possibility of explaining

\footnotetext{
${ }^{1}$ Nevertheless, one might argue that the Super-Natural SUSY has a problem related to the higgsino mass parameter $\mu$, which is generated by the GM mechanism and is proportional to the universal gaugino mass $M_{1 / 2}$, since the ratio $M_{1 / 2} / \mu$ is of order one but cannot be determined as an exact number. This problem, if it is, can be addressed in a M-theory inspired Next to MSSM (NMSSM) [51].
} 
the observed Dark Matter (DM) in terms of a relic particle produced in the early Universe through the thermal freeze-out mechanism. In Supersymmetric SMs (SSMs) with conserved $R$-parity, the Lightest Supersymmetric Particle (LSP) — such as the lightest neutralino, the gravitino, etc. - is stable and can be a dark matter candidate. However, the SSMs have in turn to fulfil the non-trivial constraints set by the DM abundance obtained from observations of the Cosmic Microwave Background (CMB). Furthermore, DM candidates have to face increasingly relevant constraints from DM searches, in particular direct detection experiments.

Another starting point of our work is the observation that string theory is one of the most promising candidates for quantum gravity. Therefore, the goal of string phenomenology is to construct the SM or SSMs from string theory with moduli stabilization and without chiral exotics, and try to make unique predictions which can probed at the LHC and other future experiments. In this article, we shall consider naturalness and dark matter phenomenology within intersecting D-brane models [52-64], where realistic SM fermion Yukawa couplings can be realized only within the Pati-Salam gauge group [65]. Three-family Pati-Salam models have been constructed systematically in Type IIA string theory on the $\mathbf{T}^{\mathbf{6}} /\left(\mathbb{Z}_{\mathbf{2}} \times \mathbb{Z}_{\mathbf{2}}\right)$ orientifold with intersecting D6-branes [58], and it was found that one model has a realistic phenomenology: the tree-level gauge coupling unification is achieved naturally around the string scale, the Pati-Salam gauge symmetry can be broken down to the SM close to the string scale, the small number of extra chiral exotic states can be decoupled via the Higgs mechanism and strong dynamics, the SM fermion masses and mixing can be accounted for, the low-energy sparticle spectra may potentially be tested at the LHC, and the observed dark matter relic density may be generated for the lightest neutralino as the LSP, and so on [66-68]. In short, this is one of the best globally consistent string models, and represents one of the few concrete string models that is phenomenologically viable from the string scale to the EWSB scale, where it features the usual spectrum of the MSSM.

The aim of the present work is to assess the naturalness of the above-mentioned Dbrane model in view of the LHC and DM constraints, and highlight spectra and other phenomenological features of the viable parameter space selected by requiring low finetuning. We base our naturalness considerations on a quantity called 'electro-weak' finetuning measure $\left(\Delta_{\mathrm{EW}}\right)$ defined as $[69,70]$

$$
\Delta_{\mathrm{EW}} \equiv \frac{\max _{a}\left|C_{a}\right|}{m_{Z}^{2} / 2}
$$

where $C_{a}$ are the terms appearing in the right-hand side of the expression

$$
\frac{m_{Z}^{2}}{2}=\frac{\left(\widetilde{m}_{H_{d}}^{2}+\Sigma_{d}\right)-\left(\widetilde{m}_{H_{u}}^{2}+\Sigma_{u}\right) \tan ^{2} \beta}{\tan ^{2} \beta-1}-|\mu|^{2},
$$

which follows from minimization of the scalar potential. Here, $\widetilde{m}_{H_{u}}^{2}$ and $\widetilde{m}_{H_{d}}^{2}$ are the SUSY breaking soft mass terms of the two Higgs doublets, and $\tan \beta$ the ratio of their vacuum expectation values (vevs), while $\mu$ is the Higgs bilinear coupling appearing in the superpotential. Explicit expressions for the quantities $\Sigma_{u, d}$, which encode 1-loop corrections 
to the tree-level potential, can be found in [71]. All quantities in eq. (1.2) are defined at low energy. For moderate to large values of $\tan \beta$, the dominant contributions to $\Delta_{\mathrm{EW}}$ stem from $\widetilde{m}_{H_{u}}^{2}$ and $\mu^{2}$. In fact, it is typically a cancellation between these two terms that ensures the correct $Z$ mass in presence of heavy superpartners (stops and gluinos, in particular), whose effect is a radiative enhancement of $\left|\widetilde{m}_{H_{u}}^{2}\right|$.

Based on what we found in previous works [40, 41], we expect to find solutions with reduced fine tuning (FT) if the Wino mass is substantially larger than the gluino mass at the unification scale. In fact, this triggers a compensation between gauge and Yukawa radiative corrections to $\widetilde{m}_{H_{u}}^{2}$, reducing its sensitivity to stop and gluino masses. This effect can be spotted from $\beta$-function of $\widetilde{m}_{H_{u}}^{2}$, which at one loop is given by

$$
16 \pi^{2} \frac{d}{d t} \widetilde{m}_{H_{u}}^{2} \approx 6 y_{t}^{2}\left[\widetilde{m}_{H_{u}}^{2}+\widetilde{m}_{Q_{3}}^{2}+\widetilde{m}_{U_{3}}^{2}+A_{t}^{2}\right]-6 g_{2}^{2} M_{2}^{2},
$$

where the hypercharge-dependent terms were omitted. The term controlled by the top Yukawa $y_{t}$ (there, $\widetilde{m}_{Q_{3}}^{2}$ and $\widetilde{m}_{U_{3}}^{2}$ are the left-handed and right-handed stop masses respectively, and $A_{t}$ the stop trilinear term) carry an opposite sign with respect to the $\mathrm{SU}(2)$ gauge term proportional to the Wino mass $M_{2}$, such that a compensation between the two terms, hence a reduced low-energy value of $\left|\widetilde{m}_{H_{u}}^{2}\right|$, is possible provided that $M_{2}>M_{3}$ (given that the gluino mass $M_{3}$ induces large positive contributions to the stop masses in the running). As we will see in the next section, this kind of non-universality of the gaugino mass terms can be easily achieved in our D-brane model, so that it will be a feature of the regions of the parameter space selected by requiring low values of $\Delta_{\mathrm{EW}}$.

The rest of the paper is organized as follows. In section 2, we review the features of the model that are relevant for our study. We describe how we preform the parameter space scan and which phenomenological constraints we impose in section 3. We present our numerical results in section 4 , and in 5 we summarize and conclude.

\section{The realistic Pati-Salam model from intersecting D6-branes}

We are going to study the realistic intersecting D6-brane model proposed in ref. [58], based on Type IIA string theory compactified on a $\mathbf{T}^{6} /\left(\mathbb{Z}_{2} \times \mathbb{Z}_{2}\right)$ orientifold, whose appealing phenomenological features have been briefly reviewed in the Introduction. Supersymmetry is broken by the F-terms of the dilaton $S$ and three complex structure moduli $U_{i}$, respectively $F^{S}$ and $F^{U_{i}}, i=1,3$. Neglecting the CP-violating phases, the resulting soft terms can be parametrized by the gravitino mass $m_{3 / 2}$, and the angles $\Theta_{1}, \Theta_{2}, \Theta_{3}$ for the complex structure moduli directions, and $\Theta_{4} \equiv \Theta_{s}$ for the dilaton one, which are related by [67]

$$
\sum_{i=1}^{4} \Theta_{i}^{2}=1 .
$$

In terms of these parameters, the soft SUSY-breaking terms at the Grand Unification (GUT) scale can be written as [67]

$$
\begin{aligned}
& M_{1}=\left(0.519 \Theta_{1}+0.346 \Theta_{2}+0.866 \Theta_{3}\right) \times m_{3 / 2}, \\
& M_{2}=\left(0.866 \Theta_{2}-0.866 \Theta_{4}\right) \times m_{3 / 2},
\end{aligned}
$$




$$
\begin{aligned}
M_{3} & =\left(0.866 \Theta_{2}+0.866 \Theta_{3}\right) \times m_{3 / 2}, \\
A_{0} & =\left(-1.111 \Theta_{1}-0.621 \Theta_{2}+0.245 \Theta_{3}-0.245 \Theta_{4}\right) \times m_{3 / 2}, \\
\widetilde{m}_{L} & =\sqrt{1.0+0.899 \Theta_{1}^{2}-0.518 \Theta_{2}^{2}-0.849 \Theta_{3}^{2}-1.418 \Theta_{4}^{2}-0.557 \Theta_{1} \Theta_{2}-0.557 \Theta_{3} \Theta_{4}} \times m_{3 / 2}, \\
\widetilde{m}_{R} & =\sqrt{1.0-1.418 \Theta_{1}^{2}-0.849 \Theta_{2}^{2}-0.518 \Theta_{3}^{2}+0.899 \Theta_{4}^{2}-0.557 \Theta_{1} \Theta_{2}-0.557 \Theta_{3} \Theta_{4}} \times m_{3 / 2}, \\
\widetilde{m}_{H_{u}} & =\widetilde{m}_{H_{d}}=\sqrt{1.0-1.5 \Theta_{3}^{2}-1.5 \Theta_{4}^{2}} \times m_{3 / 2},
\end{aligned}
$$

where $M_{1,2,3}$ are the gaugino masses, $A_{0}$ is a common trilinear term, and $\widetilde{m}_{L}$ and $\widetilde{m}_{R}$ are the soft mass terms for, respectively, the left-handed and right-handed squarks and sleptons. Notice the Pati-Salam-symmetric structure of the soft terms.

In our setup, the original gauge symmetry is $\mathrm{U}(4)_{C} \times \mathrm{U}(2)_{L} \times \mathrm{U}(2)_{R}$. The anomalies from the three global $\mathrm{U}(1) \mathrm{s}$ of $\mathrm{U}(4)_{C}, \mathrm{U}(2)_{L}$, and $\mathrm{U}(2)_{R}$ are cancelled by the generalized Green-Schwarz mechanism, and the gauge fields of these U(1)s obtain masses via the linear $B \wedge F$ couplings. Thus, the effective gauge symmetry is $\mathrm{SU}(4)_{C} \times \mathrm{SU}(2)_{L} \times \mathrm{SU}(2)_{R}$, and the three $\mathrm{U}(1) \mathrm{s}$ become global. The Higgs bilinear $\mu$ term is forbidden by the $\mathrm{U}(1)_{L}$ and $\mathrm{U}(1)_{R}$ global symmetries, and can be generated via the following dimension- 5 operator $[66,67]$

$$
W \supset \frac{y}{M_{\mathrm{St}}} S_{L} S_{R} H_{u} H_{d}
$$

where $M_{\mathrm{St}}$ is the string scale, and $S_{L}$ and $S_{R}$ are $\mathrm{SU}(2)_{L}$ and $\mathrm{SU}(2)_{R}$ anti-symmetric fields, respectively. Also, the coefficient $y$ is

$$
y \propto e^{-A},
$$

where $A$ is the area formed by the four intersections for $S_{L}, S_{R}, H_{u}$, and $H_{d}$ at classical level. In our model $[66,67]$, the vev of $S_{L}$ is close to the string scale, and the vev of $S_{R}$ is around $5 \times 10^{12} \mathrm{GeV}$. Thus, to have the $\mathrm{TeV}$-scale $\mu$ term, we require that $y$ is about $10^{-9}$, i.e., $A$ is around 20.7 in string length units. Moreover, the corresponding $B_{\mu}$ term does not have any relation with all the other supersymmetry breaking soft terms in this paper due to the superfields $S_{L}$ and $S_{R}$. Thus, for simplicity, we consider $\mu$ and $B_{\mu}$ as free parameters, which are determined by the minimization conditions for the electroweak symmetry breaking.

\section{Scanning procedure and constraints}

We employ the ISAJET 7.85 package [72] to perform random scans over the parameter space of the D-brane model presented in the previous section. Following [68], we rewrite the three independent $\Theta_{i}$ parameters that enter the soft masses in (2.2) as

$$
\begin{aligned}
& \Theta_{1}=\cos \left(\alpha_{1}\right) \cos \left(\alpha_{2}\right) \sqrt{1-\Theta_{4}^{2}}, \\
& \Theta_{2}=\sin \left(\alpha_{1}\right) \cos \left(\alpha_{2}\right) \sqrt{1-\Theta_{4}^{2}}, \\
& \Theta_{3}=\sin \left(\alpha_{2}\right) \sqrt{1-\Theta_{4}^{2}}, \\
& \text { where } \quad \alpha_{1} \equiv 2 \pi \gamma_{1}, \alpha_{2} \equiv 2 \pi \gamma_{2} .
\end{aligned}
$$


We employ the Metropolis-Hastings algorithm described in $[73,74]$ to scan over the following ranges of our parameters:

$$
\begin{aligned}
& 0 \leq \gamma_{1} \leq 1, \\
& 0 \leq \gamma_{2} \leq 1, \\
& 0 \leq \Theta_{4} \leq 1, \\
& 0 \leq m_{3 / 2} \leq 11 \mathrm{TeV}, \\
& 2 \leq \tan \beta \leq 60,
\end{aligned}
$$

For what concerns the SM parameters (e.g. the top and bottom masses), we keep the values coded into ISAJET.

We only collect data points that satisfy the requirement of a successful radiative EWSB (REWSB), i.e., a valid solution of eq. (1.2), and choose $\mu>0$. We also select the points with the lightest neutralino as the LSP. Furthermore, we consider the following constraints that we apply as specified in the next section.

LEP constraints. We impose the bounds that the LEP2 experiments set on charged sparticle masses $(\gtrsim 100 \mathrm{GeV})[75]$.

Higgs mass. The experimental combination for the Higgs mass reported by the ATLAS and CMS Collaborations is [76]

$$
m_{h}=125.09 \pm 0.21 \text { (stat.) } \pm 0.11 \text { (syst.) GeV. }
$$

Due to an estimated $2 \mathrm{GeV}$ theoretical uncertainty in the calculation of $m_{h}$ in the MSSM — see e.g. [77] — we consider the following range

$$
123 \mathrm{GeV} \leq m_{h} \leq 127 \mathrm{GeV} .
$$

$\boldsymbol{B}$-physics constraints. We use the IsaTools package [78-82] to compute the following observables and set the $2 \sigma$ constraints:

$$
\begin{aligned}
1.6 \times 10^{-9} \leq \mathrm{BR}\left(B_{s} \rightarrow \mu^{+} \mu^{-}\right) \leq 4.2 \times 10^{-9} \\
2.99 \times 10^{-4} \leq \mathrm{BR}(b \rightarrow s \gamma) \leq 3.87 \times 10^{-4} \\
0.70 \times 10^{-4} \leq \mathrm{BR}\left(B_{u} \rightarrow \tau \nu_{\tau}\right) \leq 1.5 \times 10^{-4}
\end{aligned}
$$

Electroweak fine tuning. As discussed in the Introduction, we are interested in focusing on comparably natural scenarios. Therefore, we are going to consider regions of the parameter space with a tuning better than the $\sim 1 \%$, i.e., for which the electroweak fine-tuning measure defined in eq. (1.1) satisfies

$$
\Delta_{\mathrm{EW}}<100
$$

As we have seen above, $\Delta_{\mathrm{EW}}$ is defined in terms of low-energy quantities. We verified that no additional tuning is hidden in the configurations of the high-energy parameters selected by this low-energy condition, cf. appendix A. 
LHC searches. Instead of a full recasting of the overwhelming number of searches for SUSY particles performed by the LHC Collaborations, here we only employ the latest analyses interpreted in terms of simplified models, in order to obtain approximate limits on the spectra. This approach is justified by the relative simplicity of the spectrum of our scenario, in particular for what concerns the possible light particles, such that the simplified models represent a reasonable approximation. In fact, our FT requirement in eq. (3.8) is achieved for rather heavy Winos, as explained below eq. (1.3), which radiatively increases the masses of all particles charged under $\mathrm{SU}(2)_{L}$. Furthermore, the condition (3.8) requires relatively light Higgsinos and thus neutralino LSP $(\mu \lesssim 600 \mathrm{GeV}$, see e.g. [40, 41]). This implies that the limits on gluinos and the first/second generation squarks from searches based on multi-jets and missing energy are very robust, and, due to the unified relations for the scalar masses in (2.2), affect sleptons too. In the end, only Bino, Higgsinos, righthanded stau and stop are possibly light. Based on [85-87], we consider the following condition on gluino and first/second generation squark masses

$$
\text { (a) } \quad m_{\widetilde{g}}>2 \mathrm{TeV}, \quad m_{\widetilde{q}}>2 \mathrm{TeV}
$$

which follows from the fact that the LSP is way below $1 \mathrm{TeV}$ in the scenario under consideration, and we have $m_{\widetilde{q}} \sim m_{\widetilde{g}}$ as a consequence of both the boundary conditions in (2.2) and the gluino radiative effects to squark mass terms.

Searches for two and three leptons plus missing energy [88, 89] set bounds on the electro-weak production of charged-neutral Higgsinos decaying to $W Z$ and the LSP, which we can approximately translate (cf. [90]) into the following condition

$$
\text { (b) if } m_{\widetilde{\chi}_{1}^{0}}<100 \mathrm{GeV} \Longrightarrow \mu>350 \mathrm{GeV} \text {. }
$$

Finally, searches for stops [86, 87, 91-93], including the compressed mass region, conservatively approximate to

$$
\begin{aligned}
& (c) \quad \text { if } m_{\widetilde{\chi}_{1}^{0}}<400 \mathrm{GeV} \text { and }\left(m_{\widetilde{t}_{1}}-m_{\widetilde{\chi}_{1}^{0}}\right)>100 \mathrm{GeV} \Longrightarrow m_{\widetilde{t}_{1}}>1 \mathrm{TeV}, \\
& (d) \quad \text { if } 10<\left(m_{\widetilde{t}_{1}}-m_{\widetilde{\chi}_{1}^{0}}\right)<100 \mathrm{GeV} \Longrightarrow m_{\widetilde{t}_{1}}>500 \mathrm{GeV} \\
& (e) \quad \text { if }\left(m_{\widetilde{t}_{1}}-m_{\widetilde{\chi}_{1}^{0}}\right)<5 \mathrm{GeV} \Longrightarrow m_{\widetilde{t}_{1}}>323 \mathrm{GeV} .
\end{aligned}
$$

DM searches and relic density. For the discussion on the phenomenology of neutralino DM in our scenario, we consider the following conservative range for the neutralino relic density, based on the results of [94]:

$$
0.09 \leq \Omega_{\chi} h^{2} \leq 0.14
$$

We are also going to show the impact of direct searches for DM considering the limits on the spin-independent (SI) and spin-dependent (SD) DM cross section with nuclei as presented in [95-99]. 


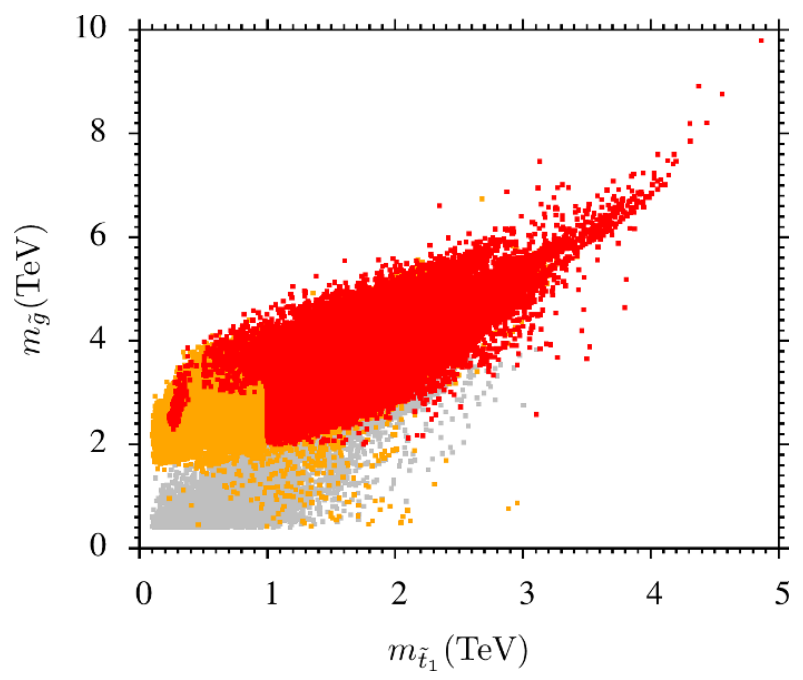

Figure 1. Result of our scan displayed on the lightest stop-gluino mass plane. All points fulfil our 'naturalness condition', $\Delta_{\mathrm{EW}} \leq 100$. Grey points satisfy the REWSB, yield a neutralino LSP and are consistent with LEP bounds. Orange points give in addition a Higgs mass in the range (3.4), while red points also satisfy $B$-physics and the LHC bounds described in section 3 .

\section{Results and discussion}

As explained above, we focus on regions of the parameter space of our D-brane scenario that corresponds to $\Delta_{\mathrm{EW}} \leq 100$, i.e., are still able to provide a relatively natural solution to the hierarchy problem. In appendix A, we show that this condition does not require tuned choices of the D-brane parameters at high energies. In figure 1, we show the resulting points on the plane of the lightest stop mass vs. the gluino mass. The grey points in the background fulfil the basic constraints discussed in the previous section. The orange points also give the correct Higgs mass, and the red ones satisfy in addition the constraints from $B$-physics observables and our approximate LHC exclusion limits, eqs. (3.9)-(3.13). This clearly shows that the LHC searches for production of strongly-interacting SUSY partners have the capability to test in part our parameter space with low tuning and have in fact excluded a corner of it already. This is in contrast to the case of models where the condition $M_{2}>M_{3}$ that reduce the sensitivity of $\widetilde{m}_{H_{u}}^{2}$ on stop and gluino masses (cf. eq. (1.3) and the discussion below it) is purely achieved by non-universal gaugino masses in gauge mediation [40]. In fact, the spectra of such models are generally beyond the reach of the LHC.

We now turn to look at the phenomenology of the lightest neutralino as DM candidate. In figure 2, we show the neutralino relic density versus to its mass, as resulting from the standard freeze-out mechanism. Grey points fulfil all constraints discussed in section 3 but are excluded by the LHC searches. Colored points satisfy such limits and highlight whether the neutralino LSP is overabundant, underabundant, or its relic density in the range of eq. (3.14). The purple points are clearly excluded by the DM relic density inferred from 


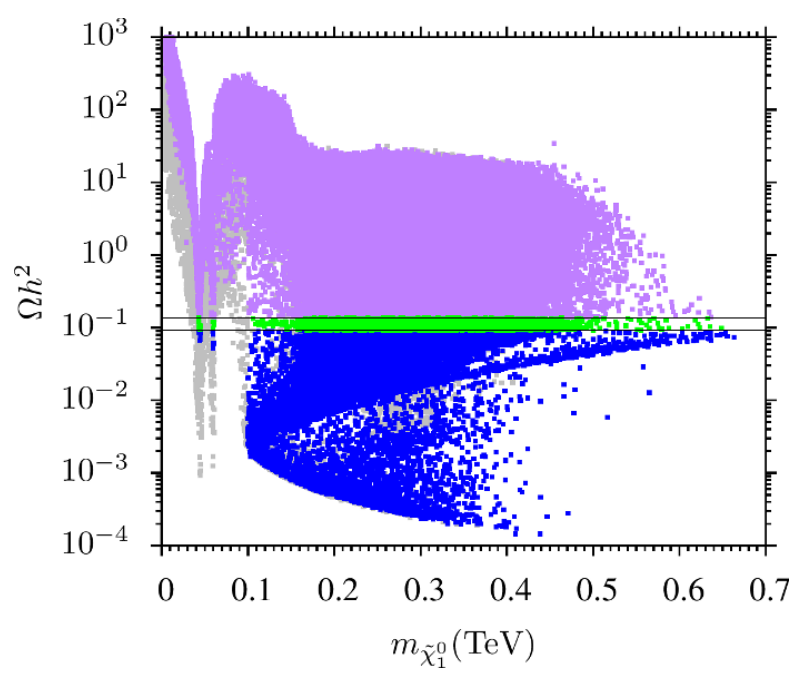

Figure 2. Neutralino relic density $\Omega_{\chi} h^{2}$ vs. its mass, $m_{\widetilde{\chi}_{1}^{0}}$. All points fulfil $\Delta_{\mathrm{EW}} \leq 100$. Grey points satisfy all the constraints discussed in section 3 except the LHC search and relic density constraints. Purple, green, and blue points are subsets of grey points representing solutions with relic density larger than, within, and lower than the range in eq. (3.14) respectively. These points also satisfy the LHC limits described in section 3.

CMB observations unless some non-standard dilution mechanism is assumed. ${ }^{2}$ On the other hand, blue points are phenomenologically viable, although they can not fully account for the observed DM, barring the case that a non-thermal production mechanism is at work. If the neutralino is lighter than about $100 \mathrm{GeV}$, the correct relic density can be achieved only on the $Z$ and $h$ resonances, $m_{\widetilde{\chi}_{1}^{0}} \approx m_{Z} / 2$ and $m_{\widetilde{\chi}_{1}^{0}} \approx m_{h} / 2$. We see from the figure that this possibility is already partially excluded by the LHC searches for heavy (Higgsino-like) neutralinos and charginos decaying $W Z$ and the LSP, as discussed in [90, 101], roughly giving the bound shown in eq. (3.10). Above $100 \mathrm{GeV}$, the LEP bounds do not forbid the LSP to be mostly Higgsino so that we can have points featuring a substantial DM underabundance. In fact, our naturalness requirement in eq. (3.8) constrains Higgsinos (and hence our neutralino LSP) to be lighter than about $600 \mathrm{GeV}$, as we can see from the figure, while a pure Higgsino LSP is underproduced unless it is as heavy as about $1.1 \mathrm{TeV}$, because of its fast annihilation modes into $\mathrm{SU}(2)_{L}$ gauge bosons.

In order to identify the neutralino annihilation or coannihilation mechanisms responsible for the results shown in figure 2, we can look at the plots of figure 3, where the same points are displayed in terms of the neutralino mass and the masses of the other particles of the model that are possibly light. In the top-left panel, we plot the chargino vs. neutralino mass, from which we can see what already mentioned above: below $m_{\widetilde{\chi}_{1}^{0}} \approx 100 \mathrm{GeV}$ the relic density constraint in eq. (3.14), can be only satisfied at the $Z$ and $h$ resonances, where

\footnotetext{
${ }^{2}$ Another option could be considering a scenario with a light axino $(\widetilde{a})$ LSP. In such a case the axino is non-thermally produced through neutralino decays, such that the resulting $\Omega_{\widetilde{a}} h^{2}$ is suppressed by a factor $m_{\tilde{a}} / m_{\tilde{\chi}_{1}^{0}}$ with respect to the neutralino density at freeze out. Nevertheless, in such a scenario, one has to check that the neutralino decay into axino is fast enough not to spoil the successful predictions of Big-Bang Nucleosynthesis (BBN). For a review, see [100].
} 

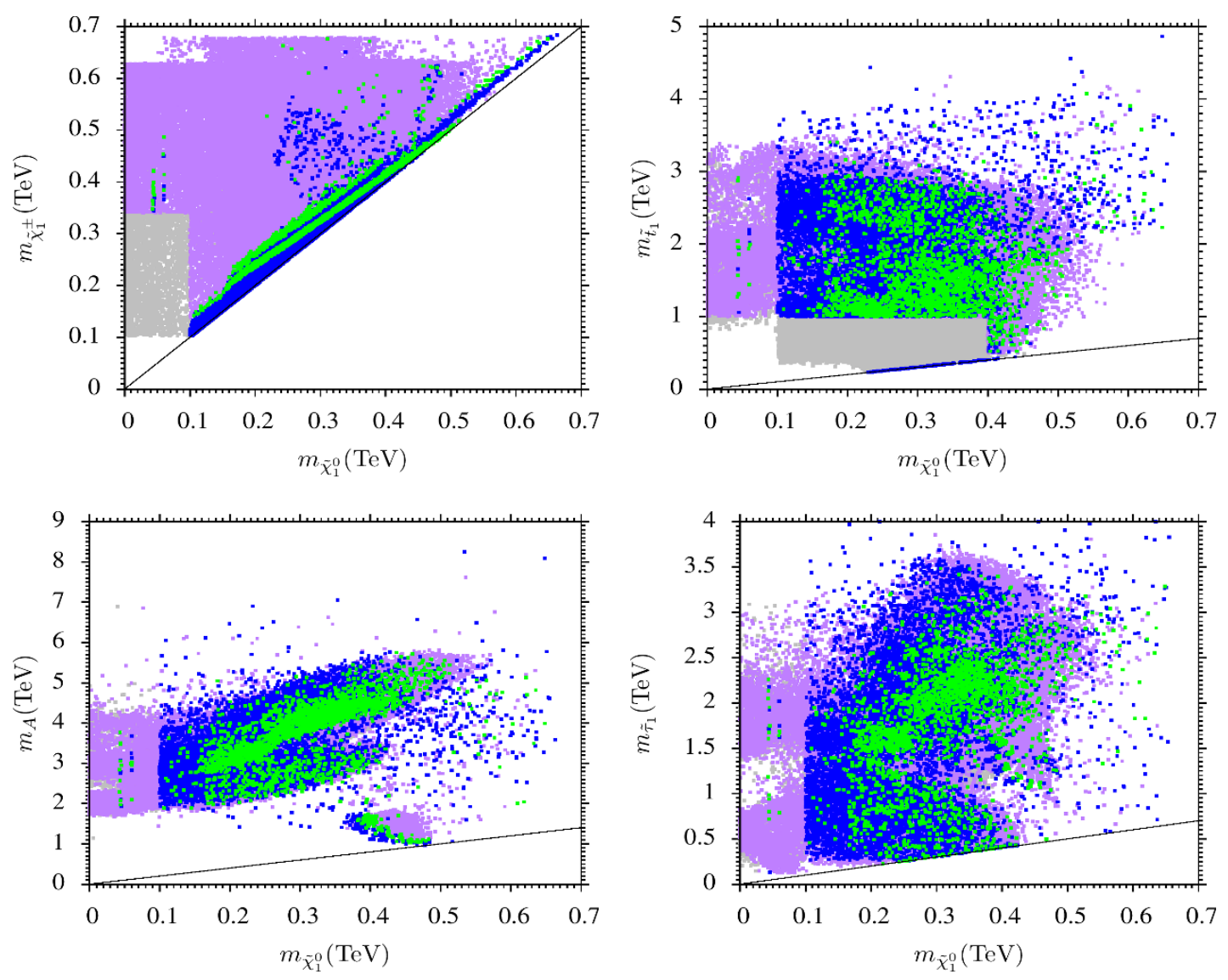

Figure 3. Points of the scan with $\Delta_{\mathrm{EW}} \leq 100$ shown on the plane of the LSP mass $m_{\widetilde{\chi}_{1}^{0}}$ and the lightest chargino mass $m_{\tilde{\chi}_{1}^{ \pm}}$(top left), stop mass $m_{\tilde{t}_{1}}$ (top right), CP-odd Higgs mass $m_{A}$ (bottom left), lightest stau mass $m_{\widetilde{\tau}_{1}}$ (bottom right). Same color code as in figure 2.

a relatively heavy Higgsino (thus chargino) is possible, since the resonant enhancement provides large annihilation rates even for relatively low Higgsino component in $\chi_{1}^{0}$. We can also see that this possibility is partially excluded by the LHC neutralino-chargino searches giving the approximate bound in eq. (3.10). Above a DM mass of $100 \mathrm{GeV}$, the underabundant blue points typically correspond to a Higgsino-like neutralino, hence neutralino and chargino are degenerate. Also, most of points with the correct relic density feature $m_{\widetilde{\chi}_{1}^{ \pm}} \gtrsim m_{\widetilde{\chi}_{1}^{0}}$, which means a large Bino-Higgsino mixing. As we will see, this possibility is now excluded by direct detection searches. There are however some green points far from the diagonal, corresponding to other annihilation mechanisms, as it is clear from the other plots in figure 3 .

In the top-right plot, where we show the stop mass, we can see that neutralino-stop coannihilations are severely constrained by our limits in eqs. (3.11-3.13). Apart from a small region with $m_{\widetilde{\chi}_{1}^{0}} \gtrsim 400 \mathrm{GeV}$, the coannihilation strips only survives for a very small mass splitting that gives in turn $\Omega_{\chi} h^{2} \ll 0.12$. The bottom row of the figure 3 shows instead that efficient annihilations through a CP-odd Higgs $A$ (bottom left) and coannihilation with the stau (bottom right) are possible in some corners of the parameter space. In particular, 

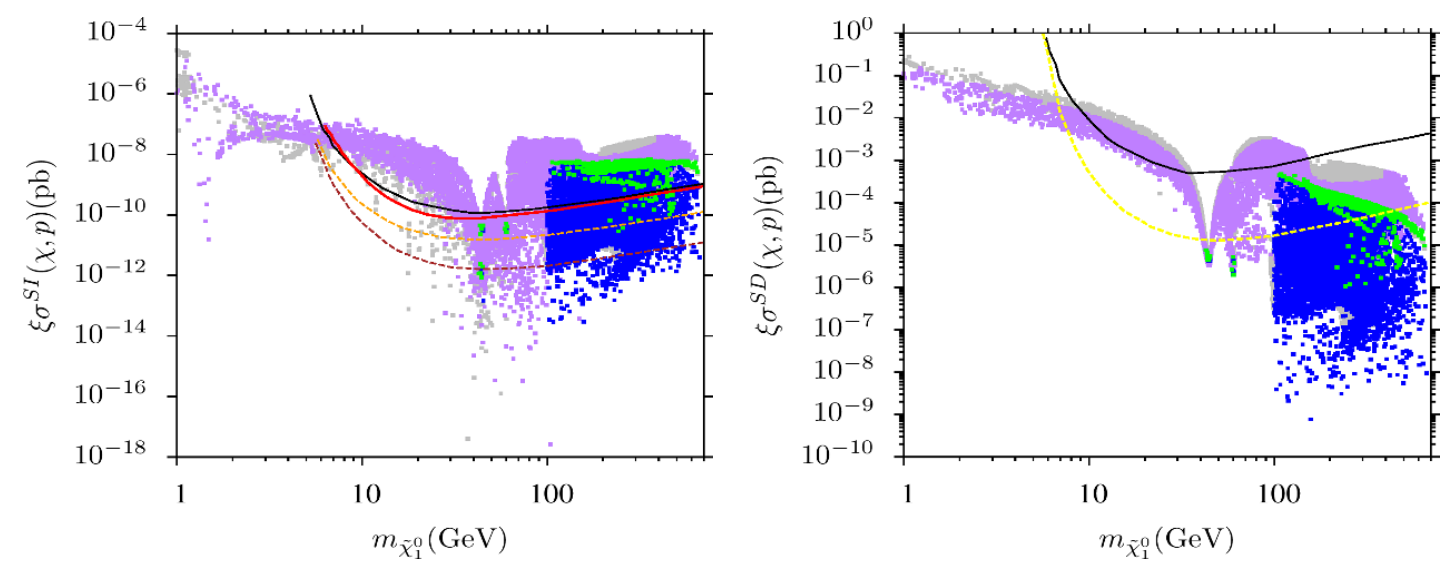

Figure 4. Rescaled spin-independent (left) and spin-dependent (right) neutralino-proton scattering cross section vs. the neutralino mass. The scaling factor is defined as $\xi \equiv \Omega_{\chi} h^{2} / 0.12$. The color code is the same as in figures 2 and 3. In the left plot, the solid black and red lines respectively represent the current LUX [95] and XENON1T [96] bounds, while the dashed orange and brown lines show the projection of future limits [97] of XENON1T with $2 t \cdot y$ exposure and XENONnT with $20 t \cdot y$ exposure, respectively. In the right plot, the black solid line is the current LUX bound [98] and the yellow dashed line represents the future LZ bound [99].

in the bottom-right plot we show that $A$ is typically heavy, but there is region where the neutralino mass is approaching the resonant condition $m_{\widetilde{\chi}_{1}^{0}} \approx m_{A} / 2$ (a solution often called ' $A$-funnel'). Large part of the plane with light $A$ and $\widetilde{\chi}_{1}^{0}$ is excluded by the interplay of the $B_{s} \rightarrow \mu^{+} \mu^{-}$and $b \rightarrow s \gamma$ constraints in combination with the Higgs mass requirement (for a discussion see e.g. [102]).

We now consider the impact of the current and future DM searches on our model, still focusing on the 'natural' regions of the parameter space as in eq. (3.8). In figure 4, we plot the spin-independent (left panel) and the spin-dependent (right panel) neutralinoproton scattering cross sections rescaled by a factor $\xi=\Omega_{\chi} h^{2} / 0.12$, which accounts for the depletion of the bounds as a consequence of a low local neutralino abundance in the cases that it can not fully account for the observed DM relic density. The present limits from direct detection experiments are shown as solid lines. As we can see, these bounds strongly affect our parameter space, especially the spin-independent one. While the $h$ and $Z$ resonances are not severely constrained at the moment, most of the (green) points compatible with the observed DM relic density (3.14) are excluded by the limits recently published by LUX and XENON1T. In particular, this is the case of the configurations with substantial Bino-Higgsino mixing, because this induces a sizable $\widetilde{\chi}_{1}^{0}-\widetilde{\chi}_{1}^{0}-h$ coupling. This scenario — some times referred to as 'well-tempered' neutralino [103] — is thus excluded in our D-brane model. For recent discussions on the direct-detection constraints on well-tempered neutralinos, see also $[104,105]$. The green points that survive the bound correspond to a Bino-like neutralino with the relic density bound fulfilled through a CP-odd Higgs exchange or stau coannihilation, as illustrated in the second row of figure 3 .

The plots in figure 4 also show that, interestingly, the future sensitivity of direct searches is capable to test almost completely our D-brane scenario with $\Delta_{\mathrm{EW}}<100$ not 


\begin{tabular}{|c|c|c|c|c|c|c|c|}
\hline & Point 1 & Point 2 & Point 3 & Point 4 & Point 5 & Point 6 & Point 7 \\
\hline$m_{L}$ & 1805 & 2048.4 & 2071.4 & 2167.7 & 5517 & 1559.4 & 4141.3 \\
\hline$m_{R}$ & 1839.7 & 1793 & 3038.5 & 1974.9 & 3383.8 & 1463.4 & 2356.7 \\
\hline$M_{1}$ & 72.02 & 105.01 & -1065.2 & 267.71 & -1608.6 & -635.52 & -607.34 \\
\hline$M_{2}$ & -3090.8 & -3057 & -4888.4 & -3158.2 & 3038.5 & -3237.7 & 3142.1 \\
\hline$M_{3}$ & -1473.2 & -1566.7 & -1479.1 & -1489.1 & 1887.7 & -1185.6 & 2305.1 \\
\hline$A_{0}$ & 1045.3 & 959.36 & 3753.5 & 658.41 & -931.66 & 2692.1 & -744.84 \\
\hline $\tan \beta$ & 17.3 & 18.8 & 54.6 & 18.3 & 44.7 & 12.8 & 46.5 \\
\hline$m_{H_{u}}=m_{H_{d}}$ & 2397.4 & 2551.8 & 2886.1 & 2519.8 & 4682.9 & 2321.6 & 4137.2 \\
\hline$\mu$ & 384 & 425 & 638 & 158 & 199 & 375 & 88 \\
\hline$\Delta_{\mathrm{EW}}$ & 37 & 43 & 99 & 32 & 14 & 63 & 88 \\
\hline$m_{h}$ & 122 & 122 & 125 & 122 & 122 & 126 & 123 \\
\hline$m_{H}$ & 2972 & 3048 & 1064 & 3053 & 3115 & 3042 & 2749 \\
\hline$m_{A}$ & 2953 & 3028 & 1057 & 3094 & 3196 & 3022 & 2730 \\
\hline$m_{H^{ \pm}}$ & 2973 & 3049 & 1069 & 3054 & 3116 & 3043 & 2750 \\
\hline$m_{\widetilde{\chi}_{1,2}^{0}}$ & $\mathbf{4 5}, 396$ & $\mathbf{6 1}, 438$ & $\mathbf{4 7 2}, 653$ & $\mathbf{1 1 7}, 166$ & $\mathbf{2 0 5}, 206$ & $\mathbf{2 7 0}, 387$ & $\mathbf{2 9 4}, 620$ \\
\hline$m_{\widetilde{\chi}_{3,4}^{0}}$ & 400,2546 & 441,2524 & 656,4042 & 186,2606 & 753,2564 & 390,2688 & 624,2638 \\
\hline$m_{\widetilde{\chi}_{1,2}^{ \pm}}$ & 375,2515 & 414,2491 & 621,4022 & $\mathbf{1 5 4}, 2572$ & $\mathbf{2 1 3}, 2531$ & 372,2684 & 637,2603 \\
\hline$m_{\widetilde{g}}$ & 3204 & 3390 & 3255 & 3248 & 4128 & 2627 & 4866 \\
\hline$m_{\widetilde{u}_{L, R}}$ & 3743,3254 & 3965,3358 & 4509,4052 & 3965,3351 & 6411,4765 & 3344,2639 & 6085,4683 \\
\hline$m_{\widetilde{t}_{1,2}}$ & 1953,3260 & 1999,3463 & 1970,3183 & 1027,2978 & 2275,5308 & $\mathbf{2 7 2}, 2774$ & 2657,5115 \\
\hline$m_{\widetilde{d}_{L, R}}$ & 3744,3257 & 3966,3361 & 4510,4051 & 3966,3354 & 64112,4753 & 3345,2639 & 6085,4682 \\
\hline$m_{\widetilde{b}_{1,2}}$ & 3156,3270 & 3241,3476 & 2695,3193 & 3244,3486 & 3788,5354 & 2536,2818 & 3785,5145 \\
\hline$m_{\widetilde{\nu}_{1,2}}$ & 2656 & 2810 & 3711 & 2944 & 5519 & 2576 & 4578 \\
\hline$m_{\widetilde{\nu}_{3}}$ & 2625 & 2773 & 3213 & 2911 & 5170 & 2565 & 4269 \\
\hline$m_{\widetilde{e}_{L, R}}$ & 2657,1838 & 2811,1790 & 3710,3064 & 2944,1975 & 5514,3425 & 2573,1479 & 4576,2356 \\
\hline$m_{\widetilde{\tau}_{1,2}}$ & 1746,2626 & 1673,2774 & 1493,3200 & 1870,2910 & 2059,5164 & 1386,2555 & $\mathbf{2 9 9}, 4265$ \\
\hline$\sigma_{\mathrm{SI}}(\mathrm{pb})$ & $4.44 \times 10^{-11}$ & $4.20 \times 10^{-11}$ & $1.48 \times 10^{-10}$ & $5.38 \times 10^{-9}$ & $1.05 \times 10^{-11}$ & $7.42 \times 10^{-10}$ & $7.44 \times 10^{-11}$ \\
\hline$\sigma_{\mathrm{SD}}(\mathrm{pb})$ & $5.53 \times 10^{-6}$ & $3.79 \times 10^{-6}$ & $3.45 \times 10^{-6}$ & $3.48 \times 10^{-4}$ & $3.49 \times 10^{-7}$ & $2.17 \times 10^{-5}$ & $1.55 \times 10^{-6}$ \\
\hline$\Omega_{\chi} h^{2}$ & 0.104 & 0.110 & 0.101 & 0.129 & 0.007 & 0.002 & 0.128 \\
\hline
\end{tabular}

Table 1. The particle spectra and properties of neutralino DM for a set of representative points for different regions of the viable parameter space. See the text in section 5 for details. The first block shows high-energy parameters defined at the GUT scale, while the others contain low-energy quantities. All quantities with mass dimension $[M]$ are in the unit of $\mathrm{GeV}$.

only for the neutralino relic density in the range of eq. (3.14), but also for most of the blue points with an underabundant neutralino due to mainly Higgsino-like LSP (for a discussion of this scenario, we refer to [106]). In summary, we see that combining our naturalness requirement with relic density constraints (that rule out the purple points) makes our model tightly constrained and in principle fully testable by DM searches, unless a substantial deviation from the standard thermal freeze-out paradigm is assumed.

\section{Summary and conclusions}

We have revisited the predicted low-energy spectra of SUSY particles in a realistic D-brane model with a particular focus on the recent LHC and DM constraints in the regions of the parameter space characterized by low levels of fine-tuning. Relatively natural solutions are possible due to the generically non-universal gaugino mass terms predicted by our model at the GUT scale, cf. the boundary conditions (2.2). In our phenomenological survey 
presented in section 4, we have found that, although several (co)annihilation modes can account for the DM abundance inferred from CMB observations, experimental constraints, in particular the LHC searches and direct DM detection, set very severe bounds on the parameter space. Interestingly, next generation direct detection experiments should be able to test the low tuning configurations of the model, as a consequence of the upper bound on the LSP mass $(\lesssim 600 \mathrm{GeV})$ set by such requiring a tuning not worse than the percent level.

We summarize our findings by showing in table 1 some points of the parameter space representative of the different regions identified and discussed in the previous section. All points feature a rather heavy spectrum and/or small mass splittings that make them not easily accessible at the LHC with the possible exception of Higgsino sector. Points 1, 2 , and 3 respectively represent the $Z$-resonance, Higgs-resonance, and $A$-funnel solutions, resulting in a neutralino relic density in the range quoted in eq. (3.14). In all three cases, the spin-independent neutralino-nucleon cross section is such that the current bounds are evaded but a signal at currently running or future direct detection experiments is expected. Point 4 is an example of the Bino-Higgsino mixed dark matter, which is already excluded by direct detection, while point 5 features a (light) mostly-Higgsino LSP, so that it is still viable because of the suppressed relic abundance. Despite that, point 5 exemplifies solutions with underabundant neutralinos in the reach of direct detection experiments, as discussed in section 4. Points 6 and 7 respectively represent solutions with efficient stopand stau-neutralino coannihilations. In the former case, the small stop-neutralino mass splitting gives as a result underabundant neutralino DM. Again, both scenarios predict a scattering cross section at levels observable at direct detection experiments.

\section{Acknowledgments}

TL was supported in part by the Projects 11475238 and 11647601 supported by National Natural Science Foundation of China, and by Key Research Program of Frontier Science, CAS. WA was supported by the CAS-TWAS Presidents Fellowship Programme. The numerical results described in this paper have been obtained via the HPC Cluster of ITP-CAS. SR would like to thank TL for warm hospitality and the Institute of Theoretical Physics, CAS, P. R. China for providing conducive atmosphere for research where part of this work has been carried out.

\section{A Tuning of the high-energy parameters}

The 'electro-weak' fine-tuning measure $\Delta_{\mathrm{EW}}$ that we employ in this paper is defined in terms of low-energy parameters, cf. eq. (1.1). Hence, one may wonder whether it fails to account for fine tuning of the high-energy parameters of our model. In this appendix, we show the output of our scan in terms of the high-energy parameters of our D-brane model that we introduced in section 2. The purpose is to show that the conditions that lead to a moderate EW tuning $\Delta_{\mathrm{EW}}$ are not met at the price of specific choices of the high-energy parameters of the theory. The result of the scan we presented in section 3 corresponding to $\Delta_{\mathrm{EW}} \leq 100$ is displayed in figure 5 for the gravitino mass $m_{3 / 2}$ versus the moduli angles $\Theta_{i}$. 

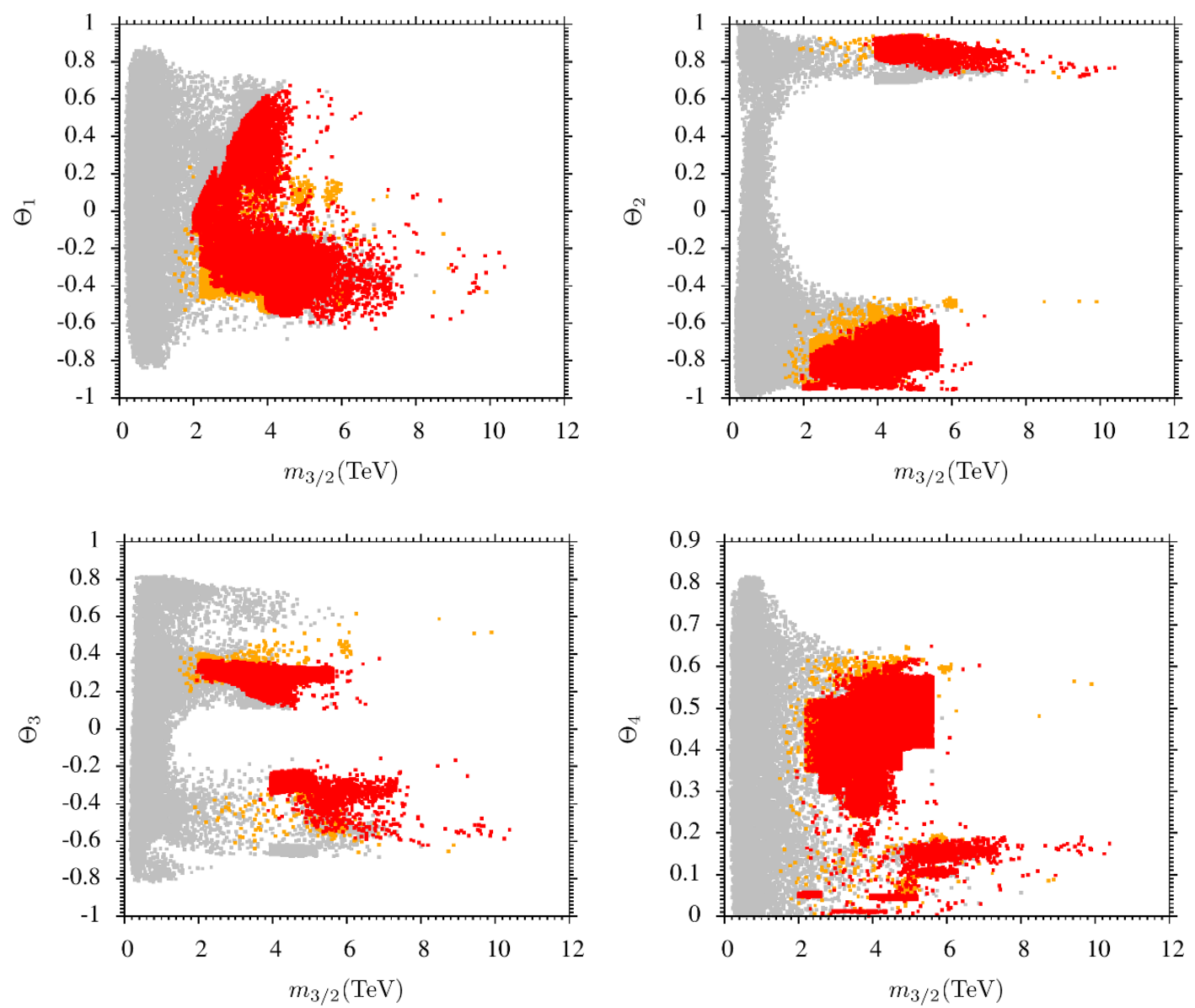

Figure 5. Low-tuning solutions $\left(\Delta_{\mathrm{EW}} \leq 100\right)$ of our scan displayed in terms of the high-energy parameters introduced in section 2 . The colors are as in figure 1.

As in figure 1, the orange points correspond to the correct Higgs mass, and the red ones satisfy in addition $B$-physics constraints and our estimated LHC exclusion limits. As we can see, the condition $\Delta_{\mathrm{EW}} \leq 100$ (which should correspond to requiring a tuning better than $1 \%$ ) together with the above phenomenological requirements certainly select certain regions on the displayed planes. However, these plots show that our solutions are rather generic, i.e., they do not require tuned choices of the high-energy parameters, surely not at the percent level. To better show this, we considered the benchmark point 1 of table 1 and performed a random variation of the moduli parameters, defined in eq. (3.1), in a $\pm 1 \%$ interval around the original values. The distribution of the resulting values of $\Delta_{\mathrm{EW}}$ is displayed in figure 6 , where the point 1 result, $\Delta_{\mathrm{EW}}=37$, is indicated by a dashed red line. As we can see, most of the solutions correspond to $\Delta_{\mathrm{EW}}$ in the same ballpark as the point 1 result. Moreover, the shift in $1 / \Delta_{\mathrm{EW}}$ is in all cases at most $\approx 1 \%$. This shows that no additional tuning (worse than the percent level) is hidden in the choice of the high-energy parameters when we impose $\Delta_{\mathrm{EW}} \leq 100$, and that $\Delta_{\mathrm{EW}}$ gives a reasonable estimate of the fine tuning of our model. 


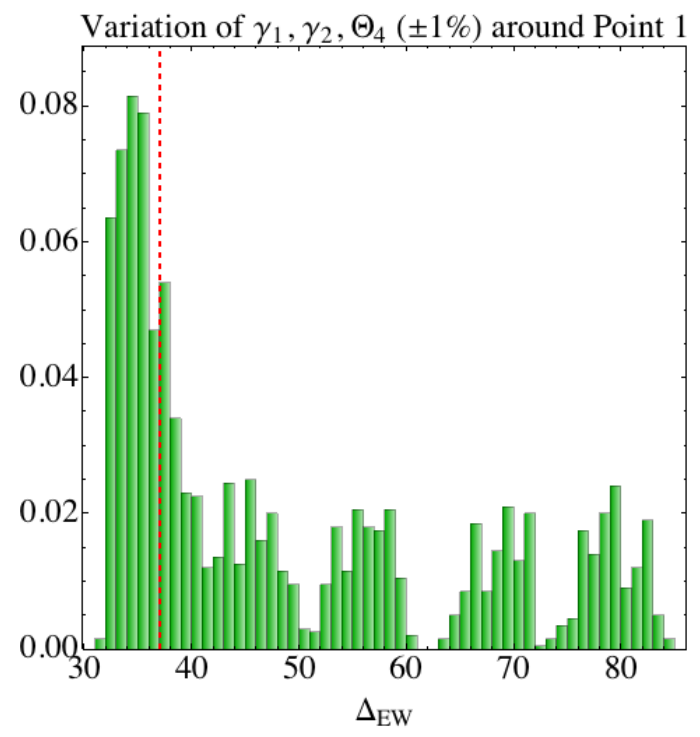

Figure 6. Frequency distribution of $\Delta_{\mathrm{EW}}$ obtained by randomly varying the moduli parameters $\alpha_{1}, \alpha_{2}, \Theta_{4}$ in the $\pm 1 \%$ interval around the values of the benchmark point 1 in table 1 . The dashed line indicates the value $\Delta_{\mathrm{EW}}=37$ obtained for point 1 .

Open Access. This article is distributed under the terms of the Creative Commons Attribution License (CC-BY 4.0), which permits any use, distribution and reproduction in any medium, provided the original author(s) and source are credited.

\section{References}

[1] H. Baer et al., Reach of the high-energy LHC for gluinos and top squarks in SUSY models with light Higgsinos, Phys. Rev. D 96 (2017) 115008 [arXiv:1708.09054] [INSPIRE].

[2] P. Fundira and A. Purves, Bayesian naturalness, simplicity and testability applied to the B-L MSSM GUT, Int. J. Mod. Phys. A 33 (2018) 1841004 [arXiv:1708.07835] [InSPIRE].

[3] J. Cao, X. Guo, Y. He, L. Shang and Y. Yue, Sneutrino DM in the NMSSM with inverse seesaw mechanism, JHEP 10 (2017) 044 [arXiv:1707.09626] [INSPIRE].

[4] B. Zhu, F. Staub and R. Ding, Naturalness and a light Z', Phys. Rev. D 96 (2017) 035038 [arXiv: 1707.03101] [INSPIRE].

[5] M. Abdughani, L. Wu and J.M. Yang, Status and prospects of light bino-higgsino dark matter in natural SUSY, Eur. Phys. J. C 78 (2018) 4 [arXiv:1705.09164] [INSPIRE].

[6] N. Liu and L. Wu, An indirect probe of the higgsino world at the CEPC, Eur. Phys. J. C 77 (2017) 868 [arXiv: 1705. 02534] [INSPIRE].

[7] H. Baer, V. Barger, M. Savoy, H. Serce and X. Tata, Superparticle phenomenology from the natural mini-landscape, JHEP 06 (2017) 101 [arXiv: 1705.01578] [INSPIRE].

[8] K.J. Bae, H. Baer and H. Serce, Prospects for axion detection in natural SUSY with mixed axion-higgsino dark matter: back to invisible?, JCAP 06 (2017) 024 [arXiv:1705.01134] [INSPIRE]. 
[9] T.-r. Liang, B. Zhu, R. Ding and T. Li, Effective natural supersymmetry from the Yukawa deflected mediations, Adv. High Energy Phys. 2017 (2017) 1585023 [arXiv: 1704.08127] [INSPIRE].

[10] C. Li, B. Zhu and T. Li, Naturalness, dark matter and the muon anomalous magnetic moment in supersymmetric extensions of the standard model with a pseudo-Dirac gluino, Nucl. Phys. B 927 (2018) 255 [arXiv: 1704.05584] [INSPIRE].

[11] P.S.B. Dev, C.M. Vila and W. Rodejohann, Naturalness in testable type-II seesaw scenarios, Nucl. Phys. B 921 (2017) 436 [arXiv: 1703.00828] [INSPIRE].

[12] H. Baer et al., What hadron collider is required to discover or falsify natural supersymmetry?, Phys. Lett. B 774 (2017) 451 [arXiv: 1702.06588] [INSPIRE].

[13] L. Delle Rose et al., Naturalness and dark matter in the supersymmetric B-L extension of the standard model, Phys. Rev. D 96 (2017) 055004 [arXiv:1702.01808] [INSPIRE].

[14] G.G. Ross, K. Schmidt-Hoberg and F. Staub, Revisiting fine-tuning in the MSSM, JHEP 03 (2017) 021 [arXiv: 1701.03480] [INSPIRE].

[15] M. van Beekveld, W. Beenakker, S. Caron, R. Peeters and R. Ruiz de Austri, Supersymmetry with Dark Matter is still natural, Phys. Rev. D 96 (2017) 035015 [arXiv:1612.06333] [INSPIRE].

[16] M. Peiro and S. Robles, Low-mass neutralino dark matter in supergravity scenarios: phenomenology and naturalness, JCAP 05 (2017) 010 [arXiv: 1612.00460] [INSPIRE].

[17] I. Gogoladze, A. Mustafayev, Q. Shafi and C.S. Un, Gauge Mediation Models with Adjoint Messengers, Phys. Rev. D 94 (2016) 075012 [arXiv:1609.02124] [INSPIRE].

[18] M. Drees and J.S. Kim, Minimal natural supersymmetry after the LHC8, Phys. Rev. D 93 (2016) 095005 [arXiv: 1511.04461] [INSPIRE].

[19] R. Ding, T. Li, F. Staub and B. Zhu, Supersoft supersymmetry, conformal sequestering and single scale supersymmetry breaking, Phys. Rev. D 93 (2016) 095028 [arXiv:1510.01328] [INSPIRE].

[20] H. Baer, V. Barger and M. Savoy, Upper bounds on sparticle masses from naturalness or how to disprove weak scale supersymmetry, Phys. Rev. D 93 (2016) 035016 [arXiv:1509.02929] [INSPIRE].

[21] B. Batell, G.F. Giudice and M. McCullough, Natural heavy supersymmetry, JHEP 12 (2015) 162 [arXiv: 1509.00834] [INSPIRE].

[22] H. Fukuda, H. Murayama, T.T. Yanagida and N. Yokozaki, Seminatural gauge mediation from product group unification, Phys. Rev. D 92 (2015) 055032 [arXiv:1508.00445] [INSPIRE].

[23] G. Bhattacharyya, T.T. Yanagida and N. Yokozaki, Focus point gauge mediation with incomplete adjoint messengers and gauge coupling unification, Phys. Lett. B 749 (2015) 82 [arXiv: 1506. 05962] [INSPIRE].

[24] S.S. AbdusSalam and L. Velasco-Sevilla, Where to look for natural supersymmetry, Phys. Rev. D 94 (2016) 035026 [arXiv: 1506.02499] [InSPIRE].

[25] D. Barducci, A. Belyaev, A.K.M. Bharucha, W. Porod and V. Sanz, Uncovering natural supersymmetry via the interplay between the LHC and Direct Dark Matter Detection, JHEP 07 (2015) 066 [arXiv: 1504.02472] [INSPIRE].

[26] T. Cohen, J. Kearney and M. Luty, Natural Supersymmetry without Light Higgsinos, Phys. Rev. D 91 (2015) 075004 [arXiv:1501.01962] [INSPIRE]. 
[27] J. Fan, M. Reece and L.-T. Wang, Precision natural SUSY at CEPC, FCC-ee and ILC, JHEP 08 (2015) 152 [arXiv: 1412.3107] [INSPIRE].

[28] S. Dimopoulos, K. Howe and J. March-Russell, Maximally natural supersymmetry, Phys. Rev. Lett. 113 (2014) 111802 [arXiv:1404.7554] [INSPIRE].

[29] E. Hardy, Heavy superpartners with less tuning from hidden sector renormalisation, JHEP 03 (2014) 069 [arXiv: 1311.2944] [INSPIRE].

[30] I. Gogoladze, F. Nasir and Q. Shafi, $\mathrm{SO}(10)$ as a framework for natural supersymmetry, JHEP 11 (2013) 173 [arXiv:1306.5699] [INSPIRE].

[31] G.D. Kribs, A. Martin and A. Menon, Natural supersymmetry and implications for Higgs physics, Phys. Rev. D 88 (2013) 035025 [arXiv: 1305.1313] [INSPIRE].

[32] A. Kaminska, G.G. Ross and K. Schmidt-Hoberg, Non-universal gaugino masses and fine tuning implications for SUSY searches in the MSSM and the GNMSSM, JHEP 11 (2013) 209 [arXiv: 1308.4168] [INSPIRE].

[33] C. Boehm, P.S.B. Dev, A. Mazumdar and E. Pukartas, Naturalness of light neutralino dark matter in pMSSM after LHC, XENON100 and Planck Data, JHEP 06 (2013) 113 [arXiv: 1303.5386] [INSPIRE].

[34] F. Brümmer, M. Ibe and T.T. Yanagida, Focus point gauge mediation in product group unification, Phys. Lett. B 726 (2013) 364 [arXiv: 1303.1622] [INSPIRE].

[35] E. Hardy and J. March-Russell, Retrofitted natural supersymmetry from a U(1), JHEP 05 (2013) 120 [arXiv:1302.5423] [INSPIRE].

[36] I. Gogoladze, F. Nasir and Q. Shafi, Non-universal gaugino masses and natural supersymmetry, Int. J. Mod. Phys. A 28 (2013) 1350046 [arXiv:1212.2593] [INSPIRE].

[37] S. Antusch et al., Naturalness of the non-universal MSSM in the light of the recent Higgs results, JHEP 01 (2013) 187 [arXiv:1207.7236] [INSPIRE].

[38] F. Brummer and W. Buchmüller, The Fermi scale as a focus point of high-scale gauge mediation, JHEP 05 (2012) 006 [arXiv:1201.4338] [INSPIRE].

[39] D. Horton and G.G. Ross, Naturalness and focus points with non-universal gaugino masses, Nucl. Phys. B 830 (2010) 221 [arXiv:0908.0857] [INSPIRE].

[40] L. Calibbi, T. Li, A. Mustafayev and S. Raza, Improving naturalness in gauge mediation with nonunified messenger sectors, Phys. Rev. D 93 (2016) 115018 [arXiv:1603.06720] [INSPIRE].

[41] W. Ahmed, L. Calibbi, T. Li, A. Mustafayev and S. Raza, Low fine tuning in Yukawa-deflected gauge mediation, Phys. Rev. D 95 (2017) 095031 [arXiv:1612.07125] [INSPIRE].

[42] T. Leggett et al., No naturalness or fine-tuning problems from no-scale supergravity, arXiv:1403.3099 [INSPIRE].

[43] T. Leggett et al., Confronting Electroweak Fine-tuning with No-Scale Supergravity, Phys. Lett. B 740 (2015) 66 [arXiv:1408.4459] [INSPIRE].

[44] G. Du, T. Li, D.V. Nanopoulos and S. Raza, Super-natural MSSM, Phys. Rev. D 92 (2015) 025038 [arXiv: 1502.06893] [INSPIRE].

[45] E. Cremmer, S. Ferrara, C. Kounnas and D.V. Nanopoulos, Naturally vanishing cosmological constant in $N=1$ supergravity, Phys. Lett. 133B (1983) 61 [INSPIRE].

[46] J.R. Ellis, A.B. Lahanas, D.V. Nanopoulos and K. Tamvakis, No-scale supersymmetric standard model, Phys. Lett. 134B (1984) 429 [INSPIRE]. 
[47] J.R. Ellis, C. Kounnas and D.V. Nanopoulos, Phenomenological SU $(1,1)$ supergravity, Nucl. Phys. B 241 (1984) 406 [INSPIRE].

[48] J.R. Ellis, C. Kounnas and D.V. Nanopoulos, No scale supersymmetric guts, Nucl. Phys. B 247 (1984) 373 [INSPIRE].

[49] A.B. Lahanas and D.V. Nanopoulos, The road to no scale supergravity, Phys. Rept. 145 (1987) 1 [INSPIRE].

[50] G.F. Giudice and A. Masiero, A natural solution to the $\mu$ problem in supergravity theories, Phys. Lett. B 206 (1988) 480 [INSPIRE].

[51] T. Li, S. Raza and X.-C. Wang, Supernatural supersymmetry and its classic example: M-theory inspired NMSSM, Phys. Rev. D 93 (2016) 115014 [arXiv:1510.06851] [inSPIRE].

[52] M. Berkooz, M.R. Douglas and R.G. Leigh, Branes intersecting at angles, Nucl. Phys. B 480 (1996) 265 [hep-th/9606139] [INSPIRE].

[53] L.E. Ibáñez, F. Marchesano and R. Rabadán, Getting just the standard model at intersecting branes, JHEP 11 (2001) 002 [hep-th/0105155] [INSPIRE].

[54] R. Blumenhagen, B. Körs, D. Lüst and T. Ott, The standard model from stable intersecting brane world orbifolds, Nucl. Phys. B 616 (2001) 3 [hep-th/0107138] [INSPIRE].

[55] M. Cvetič, G. Shiu and A.M. Uranga, Three family supersymmetric standard-like models from intersecting brane worlds, Phys. Rev. Lett. 87 (2001) 201801 [hep-th/0107143] [INSPIRE].

[56] M. Cvetič, G. Shiu and A.M. Uranga, Chiral four-dimensional $N=1$ supersymmetric type $2 A$ orientifolds from intersecting D6 branes, Nucl. Phys. B 615 (2001) 3 [hep-th/0107166] [INSPIRE].

[57] M. Cvetič, I. Papadimitriou and G. Shiu, Supersymmetric three family SU(5) grand unified models from type IIA orientifolds with intersecting D6-branes, Nucl. Phys. B 659 (2003) 193 [Erratum ibid. B 696 (2004) 298] [hep-th/0212177] [INSPIRE].

[58] M. Cvetič, T. Li and T. Liu, Supersymmetric patiSalam models from intersecting D6-branes: a road to the standard model, Nucl. Phys. B 698 (2004) 163 [hep-th/0403061] [INSPIRE].

[59] M. Cvetič, P. Langacker, T.-j. Li and T. Liu, D6-brane splitting on type IIA orientifolds, Nucl. Phys. B 709 (2005) 241 [hep-th/0407178] [INSPIRE].

[60] M. Cvetič, T. Li and T. Liu, Standard-like models as type IIB flux vacua, Phys. Rev. D 71 (2005) 106008 [hep-th/0501041] [INSPIRE].

[61] C.M. Chen et al., A supersymmetric flipped SU(5) intersecting brane world, Phys. Lett. B 611 (2005) 156 [hep-th/0501182] [INSPIRE].

[62] C.M. Chen et al., A k-theory anomaly free supersymmetric flipped $\mathrm{SU}(5)$ model from intersecting branes, Phys. Lett. B 625 (2005) 96 [hep-th/0507232] [INSPIRE].

[63] C.-M. Chen, T. Li and D.V. Nanopoulos, Standard-like model building on Type II orientifolds, Nucl. Phys. B 732 (2006) 224 [hep-th/0509059] [INSPIRE].

[64] R. Blumenhagen, M. Cvetič, P. Langacker and G. Shiu, Toward realistic intersecting D-brane models, Ann. Rev. Nucl. Part. Sci. 55 (2005) 71 [hep-th/0502005] [inSPIRE].

[65] J.C. Pati and A. Salam, Lepton number as the fourth color, Phys. Rev. D 10 (1974) 275 [Erratum ibid. D 11 (1975) 703] [INSPIRE].

[66] C.-M. Chen, T. Li, V.E. Mayes and D.V. Nanopoulos, A realistic world from intersecting D6-branes, Phys. Lett. B 665 (2008) 267 [hep-th/0703280] [INSPIRE]. 
[67] C.-M. Chen, T. Li, V.E. Mayes and D.V. Nanopoulos, Towards realistic supersymmetric spectra and Yukawa textures from intersecting branes, Phys. Rev. D 77 (2008) 125023 [arXiv:0711.0396] [INSPIRE].

[68] T. Li, D.V. Nanopoulos, S. Raza and X.-C. Wang, A Realistic Intersecting D6-Brane Model after the First LHC Run, JHEP 08 (2014) 128 [arXiv: 1406.5574] [INSPIRE].

[69] H. Baer et al., Radiative natural SUSY with a 125 GeV Higgs boson, Phys. Rev. Lett. 109 (2012) 161802 [arXiv:1207.3343] [INSPIRE].

[70] H. Baer et al., Post-LHC7 fine-tuning in the minimal supergravity/CMSSM model with a 125 GeV Higgs boson, Phys. Rev. D 87 (2013) 035017 [arXiv:1210.3019] [INSPIRE].

[71] H. Baer et al., Radiative natural supersymmetry: Reconciling electroweak fine-tuning and the Higgs boson mass, Phys. Rev. D 87 (2013) 115028 [arXiv:1212.2655] [INSPIRE].

[72] H. Baer, F.E. Paige, S.D. Protopopescu and X. Tata, ISAJET 7.48: a Monte Carlo event generator for $p p, \bar{p} p$ and $e^{+} e^{-}$reactions, hep-ph/0001086 [INSPIRE].

[73] G. Bélanger, F. Boudjema, A. Pukhov and R.K. Singh, Constraining the MSSM with universal gaugino masses and implication for searches at the LHC, JHEP 11 (2009) 026 [arXiv:0906.5048] [inSPIRE].

[74] H. Baer, S. Kraml, S. Sekmen and H. Summy, Dark matter allowed scenarios for Yukawa-unified SO(10) SUSY GUTs, JHEP 03 (2008) 056 [arXiv:0801.1831] [InSPIRE].

[75] Particle Data Group collaboration, C. Patrignani et al., Review of particle physics, Chin. Phys. C 40 (2016) 100001 [inSPIRE].

[76] ATLAS, CMS collaboration, G. Aad et al., Measurements of the Higgs boson production and decay rates and constraints on its couplings from a combined ATLAS and CMS analysis of the LHC pp collision data at $\sqrt{s}=7$ and 8 TeV, JHEP 08 (2016) 045 [arXiv: 1606.02266] [INSPIRE].

[77] B.C. Allanach et al., Precise determination of the neutral Higgs boson masses in the MSSM, JHEP 09 (2004) 044 [hep-ph/0406166] [INSPIRE].

[78] H. Baer, M. Brhlik, C.-h. Chen and X. Tata, Signals for the minimal gauge mediated supersymmetry breaking model at the Fermilab Tevatron collider, Phys. Rev. D 55 (1997) 4463 [hep-ph/9610358] [INSPIRE].

[79] H. Baer, M. Brhlik, D. Castano and X. Tata, $b \rightarrow$ sgamma constraints on the minimal supergravity model with large $\tan \beta$, Phys. Rev. D 58 (1998) 015007 [hep-ph/9712305] [INSPIRE].

[80] K.S. Babu and C.F. Kolda, Higgs mediated $B^{0} \rightarrow \mu^{+} \mu^{-}$in minimal supersymmetry, Phys. Rev. Lett. 84 (2000) 228 [hep-ph/9909476] [INSPIRE].

[81] A. Dedes, H.K. Dreiner and U. Nierste, Correlation of $B_{s} \rightarrow \mu^{+} \mu^{-}$and $(g-2)_{\mu}$ in minimal supergravity, Phys. Rev. Lett. 87 (2001) 251804 [hep-ph/0108037] [INSPIRE].

[82] J.K. Mizukoshi, X. Tata and Y. Wang, Higgs mediated leptonic decays of $B_{s}$ and $B_{d}$ mesons as probes of supersymmetry, Phys. Rev. D 66 (2002) 115003 [hep-ph/0208078] [INSPIRE].

[83] LHC and CMS collaborations, Observation of the rare $B_{s}^{0} \rightarrow \mu^{+} \mu^{-}$decay from the combined analysis of CMS and LHCb data, Nature $\mathbf{5 2 2}$ (2015) 68 [arXiv:1411.4413] [INSPIRE].

[84] Heavy Flavor Averaging Group (HFAG) collaboration, Y. Amhis et al., Averages of b-hadron, c-hadron and $\tau$-lepton properties as of summer 2014, arXiv:1412.7515 [INSPIRE].

[85] ATLAS collaboration, Search for squarks and gluinos in final states with jets and missing transverse momentum using $36 \mathrm{fb}^{-1}$ of $\sqrt{\mathrm{s}}=13 \mathrm{TeV}$ pp collision data with the ATLAS detector, ATLAS-CONF-2017-022 (2017). 
[86] CMS collaboration, Search for supersymmetry in multijet events with missing transverse momentum in proton-proton collisions at 13 TeV, Phys. Rev. D 96 (2017) 032003 [arXiv: 1704.07781] [INSPIRE].

[87] CMS collaboration, Search for new phenomena with the $M_{\mathrm{T} 2}$ variable in the all-hadronic final state produced in proton-proton collisions at $\sqrt{s}=13$ TeV, Eur. Phys. J. C 77 (2017) 710 [arXiv: 1705. 04650] [INSPIRE].

[88] CMS collaboration, Search for electroweak production of charginos and neutralinos in multilepton final states in pp collision data at $\sqrt{s}=13$ TeV, CMS-PAS-SUS-16-039 (2016).

[89] ATLAS collaboration, Search for electroweak production of supersymmetric particles in the two and three lepton final state at $\sqrt{s}=13$ TeV with the ATLAS detector, ATLAS-CONF-2017-039 (2017).

[90] W. Ahmed et al., Status of natural supersymmetry from the GmSUGRA in light of the current LHC Run-2 and LUX data, arXiv:1709.06371 [INSPIRE].

[91] CMS collaboration, Search for direct top squark pair production in the all-hadronic final state in proton-proton collisions at $\sqrt{s}=13$ TeV, CMS-PAS-SUS-16-049 (2017).

[92] ATLAS collaboration, Search for a scalar partner of the top quark in the jets+ETmiss final state at $\sqrt{s}=13$ TeV with the ATLAS detector, ATLAS-CONF-2017-020 (2017).

[93] ATLAS collaboration, Search for new phenomena in final states with an energetic jet and large missing transverse momentum in pp collisions at $\sqrt{s}=13$ TeV using the ATLAS detector, Phys. Rev. D 94 (2016) 032005 [arXiv:1604.07773] [INSPIRE].

[94] Planck collaboration, P.A.R. Ade et al., Planck 2015 results. XIII. Cosmological parameters, Astron. Astrophys. 594 (2016) A13 [arXiv:1502.01589] [InSPIRE].

[95] LUX collaboration, D.S. Akerib et al., Results from a search for dark matter in the complete LUX exposure, Phys. Rev. Lett. 118 (2017) 021303 [arXiv:1608.07648] [InSPIRE].

[96] XENON collaboration, E. Aprile et al., First dark matter search results from the XENON1T experiment, Phys. Rev. Lett. 119 (2017) 181301 [arXiv:1705.06655] [INSPIRE].

[97] XENON collaboration, E. Aprile et al., Physics reach of the XENON1T dark matter experiment, JCAP 04 (2016) 027 [arXiv: 1512.07501] [INSPIRE].

[98] LUX collaboration, D.S. Akerib et al., Limits on spin-dependent WIMP-nucleon cross section obtained from the complete LUX exposure, Phys. Rev. Lett. 118 (2017) 251302 [arXiv: 1705.03380] [INSPIRE].

[99] LUX collaboration, D.S. Akerib et al., Results on the spin-dependent scattering of weakly interacting massive particles on nucleons from the run 3 data of the LUX experiment, Phys. Rev. Lett. 116 (2016) 161302 [arXiv:1602.03489] [INSPIRE].

[100] K.-Y. Choi, J.E. Kim and L. Roszkowski, Review of axino dark matter, J. Korean Phys. Soc. 63 (2013) 1685 [arXiv:1307.3330] [INSPIRE].

[101] L. Calibbi, J.M. Lindert, T. Ota and Y. Takanishi, LHC tests of light neutralino dark matter without light sfermions, JHEP 11 (2014) 106 [arXiv: 1410.5730] [INSPIRE].

[102] L. Calibbi, T. Ota and Y. Takanishi, Light neutralino in the MSSM: a playground for dark matter, flavor physics and collider experiments, JHEP 07 (2011) 013 [arXiv: 1104.1134] [INSPIRE].

[103] N. Arkani-Hamed, A. Delgado and G.F. Giudice, The well-tempered neutralino, Nucl. Phys. B 741 (2006) 108 [hep-ph/0601041] [INSPIRE].

[104] M. Badziak, M. Olechowski and P. Szczerbiak, Is well-tempered neutralino in MSSM still alive after 2016 LUX results?, Phys. Lett. B 770 (2017) 226 [arXiv:1701.05869] [INSPIRE]. 
[105] S. Profumo, T. Stefaniak and L. Stephenson Haskins, The not-so-well tempered neutralino, Phys. Rev. D 96 (2017) 055018 [arXiv:1706. 08537] [INSPIRE].

[106] M. Badziak et al., Detecting underabundant neutralinos, JHEP 11 (2015) 053 [arXiv: 1506.07177] [INSPIRE]. 